\title{
Cardiac Yolk Sac Tumor
}

National Cancer Institute

\section{Source}

National Cancer Institute. Cardiac Yolk Sac Tumor. NCI Thesaurus. Code C147006.

A yolk sac tumor that arises within the myocardium or cardiac chambers. 